\title{
The perplexity of malignant ovarian mass in cystadenofibroma: a lesson learnt
}

\author{
Priyanka Kathuria*, Garima Yadav, Pratibha Singh
}

Department of Obstetrics and Gynecology, All India Institue of Medical Sciences, Jodhpur, Rajasthan, India

Received: 03 December 2018

Accepted: 14 December 2018

\section{*Correspondence:}

Dr. Priyanka Kathuria,

E-mail: dr.priyankathuria@gmail.com

Copyright: $($ ) the author(s), publisher and licensee Medip Academy. This is an open-access article distributed under the terms of the Creative Commons Attribution Non-Commercial License, which permits unrestricted non-commercial use, distribution, and reproduction in any medium, provided the original work is properly cited.

\begin{abstract}
The aim of this study is to report a rare case of ovarian cystadenofibroma with even scarcely observed mucinous type histopathological variant and to discuss the modalities which can contribute to prevent misdiagnosing it as a malignant tumor that may inappropriately lead to aggressive surgical interventions. A 40-year-old female presented with pain in lower abdomen and dysmenorrhea of 3 months duration and a large right adnexal mass which had all the features suggestive of malignancy in pre-operative investigations and even on gross overview and cut section. On the contrary histopathology reports confirmed its benign nature and reported it as mucinous cystadenofibroma. Role of Preoperative MRI and intraoperative frozen section cannot be overemphasized in distinguishing ovarian cystadenofibroma from malignant tumors, which can avoid an unnecessary extensive surgery.
\end{abstract}

Keywords: Benign, Cystadenofibroma, Malignant, Ovary

\section{INTRODUCTION}

Ovarian cystadenofibroma is a rare benign tumor of the ovary. It originates from the germinal lining and stroma of the ovary with an incidence of approximately $1.7 \%$ of all benign ovarian neoplasms. 1 It may be seen in women aged 15-65 years, but mostly presents in the fourth and fifth decades of life. ${ }^{2}$ The mucinous variety presents in a little younger age group. The exact cause of Benign Cystadenofibroma of Ovary is unknown, and these are considered to occur spontaneously. In past, a subset of women exposed to antenatal diethylstilbestrol were deemed predisposed to develop such tumors and they presented at an earlier age. ${ }^{3}$

It is a slow-growing epithelial tumor which may present with signs and symptoms such as to result in misdiagnoses as a malignant tumor. The common signs being abdominal pain, abdominal lump, distension, dyspepsia and vaginal bleeding. Many such tumors may not show any signs or symptoms at all and may be detected incidentally during an ultrasound done for some other reason. Preliminary imaging including USG or CT may also not prove valuable in differentiating them from malignant tumors. Although MRI showing low-signal intensity on $\mathrm{T} 2 \mathrm{~W}$ images owing to the fibrous nature of the lesion may help in suspecting the condition preoperatively.

Most of the reported adenofibromas are of serous type while mucinous, endometrioid, clear cell and mixed types being even rarer. ${ }^{4}$ We hereby describe a case of a $40-$ year-old woman with an ovarian cyst which turned out to be mucinous cystadenofibroma on histopathology.

\section{CASE REPORT}

A 40-year-old female para 3 presented with complaints of pain in lower abdomen and irregular frequent periods associated with dysmenorrhea for 3 months. Her previous menstrual cycles were regular with no significant medical or family history. 
At the time of presentation, patient's general condition was good, her per abdomen examination revealed a large cystic globular mass with size around 22-week gravid uterus. The lump was firm, had irregular margins, was mobile sideways and the lower limit of the mass could be reached. On per speculum examination cervix and vagina appeared healthy and a cervical cytology sample was taken as a part of routine screening. Per vaginal examination revealed the same mass, felt more in the anterior fornix and pushing the uterus posteriorly. The mass was firm in consistency, had irregular margins and did not move with the movement of uterus. Per rectal examination showed free rectal mucosa with no nodularity in the pouch of douglas.
Upon comprehensive medical testing, an ovarian mass was detected. CT report showed a mass centered more on right side which was cystic with solid areas with multiple enhancing septations. The mass was displacing adjacent bowel loops.

The mass measured $18 \times 16 \times 11 \mathrm{~cm}$ on CT. However, there was no evidence of invasion. Right ovary was not seen separately. Left ovary was normal. Minimal free fluid seen in pouch of douglas.

Multiple enlarged and necrotic lymph nodes were seen in paraaortic $(13 \times 10 \mathrm{~mm})$, aortocaval $(13 \times 12 \mathrm{~mm} 0$, right iliac (20x7mm) and in $\operatorname{RIF}(12 \times 10 \mathrm{~mm})$ regions.

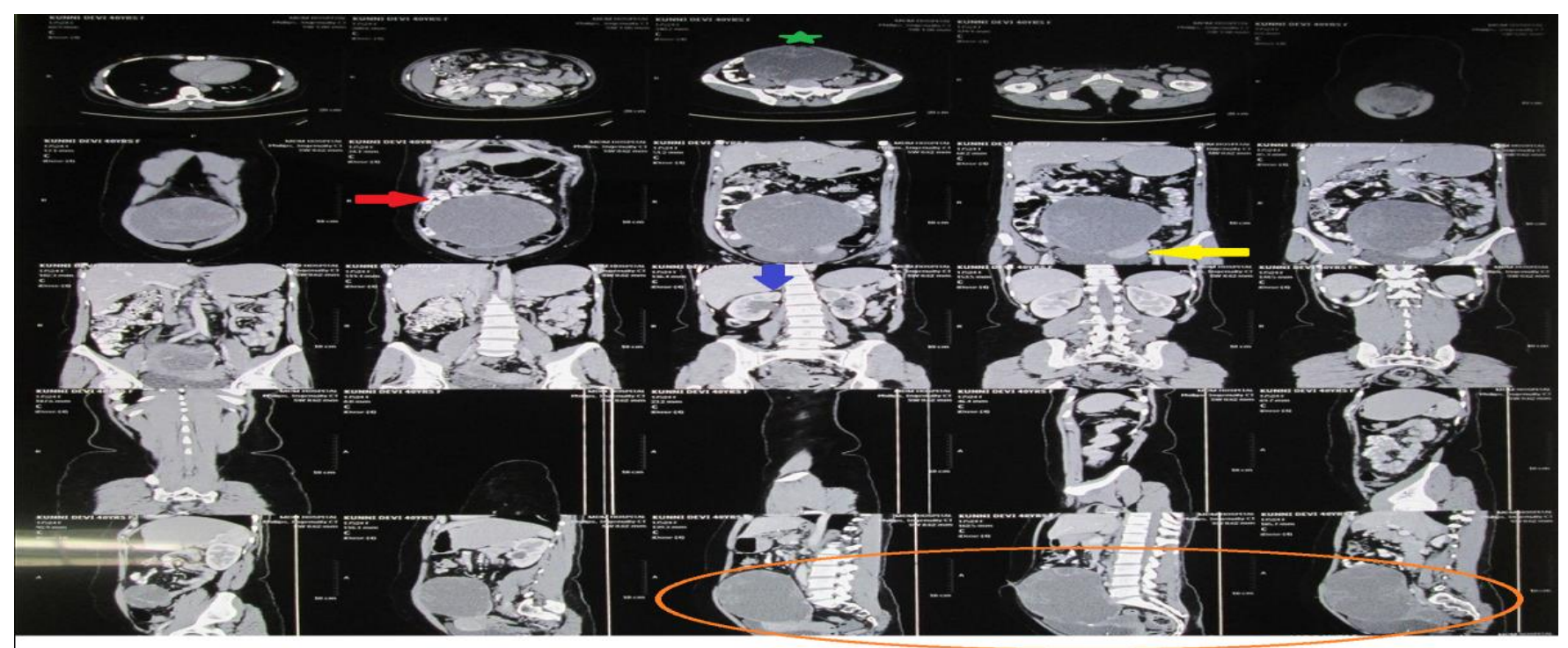

Figure1: CT film showing the mass with multiple enhancing septations (Green Star) with displaced bowel loops (Red arrow pointing right) and uterus (Yellow arrow pointing left). Right kidney shows mild hydronephrosis (blue arrow pointing downwards). Last three images exhibit the extent of the mass (orange ellipse).

Fat planes with adjacent structures were maintained. Right kidney showed mild hydronephrosis. Upper abdomen was normal (Figure 1).

All her routine pre-anesthetic work up was normal. Serum concentrations of the tumor markers CA-125 was $22.64 \mathrm{U} / \mathrm{ml}$, AFP was $3.21 \mathrm{ng} / \mathrm{ml}$ and CEA was 0.832 $\mathrm{ng} / \mathrm{ml}$. Sonography (transabdominal + transvaginal) showed a normal uterus and a right ovarian mass approximately $10 \times 15 \times 12 \mathrm{~cm}$ in size with a volume of around $1400 \mathrm{cc}$. It was thick walled with a large cystic area and multiple thick septations and few small solid areas. The left adnexa was normal.

The patient underwent Staging laparotomy under GA supported by epidural analgesia. A low midline incision was given which was later extended supraumbilically. The uterus was of normal size.

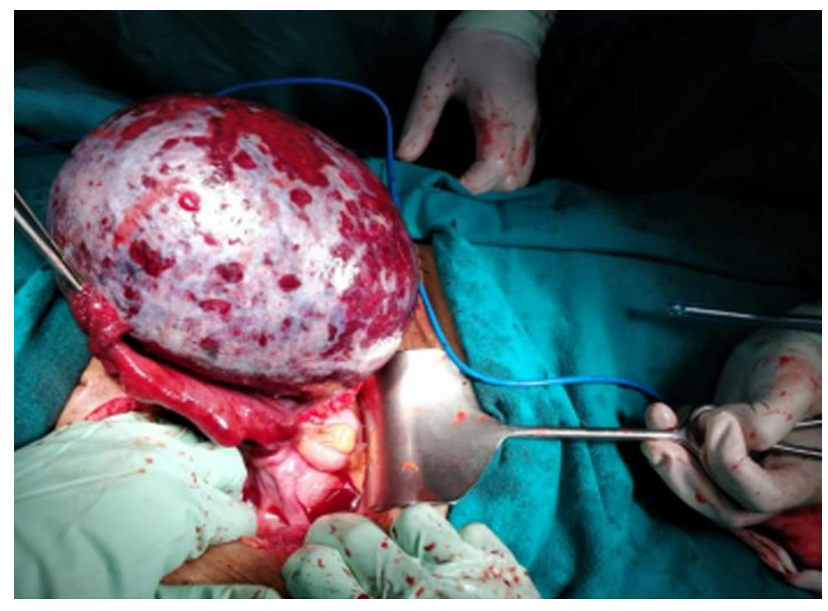

Figure 2: Intraoperative image after delivering the mass. 
Right ovarian cyst approximately $15 \times 12 \times 10 \mathrm{~cm}$ size was observed (Figure 2). The mass was very hard with a few cystic areas and a relatively smooth surface.

While delivering the cyst, a small amount of mucinous fluid was drained out of it. The mass had solid areas with interspersed cystic areas filled with mucinous fluid and a few hemorrhages (Figure 3). Hence, suspecting malignancy, infracolicomentectomy and lymph node dissection was carried out along with multiple peritoneal biopsies and appendectomy. Later, the histopathological report confirmed it to be mucinous cystadenofibroma, following which, the patient was discharged on 5th postoperative day in a stable condition. The patient did not require any additional treatment beyond regular checkups.

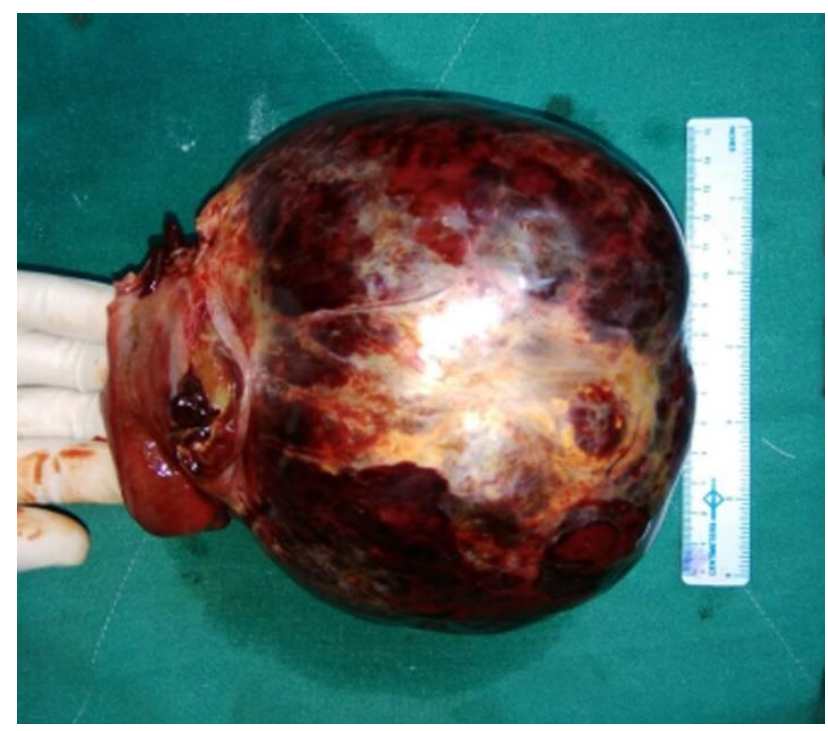

Figure 3: Gross specimen with scale.

\section{DISCUSSION}

Although classified as an epithelial tumour, adenofibroma (and a cystadenofibroma) contains both epithelial and stromal components. The ratio of the epithelial and stromal components and the amount of secretory function of the epithelial component dictates the nomenclature and gross appearance of the tumor (solid, semisolid or liquid state).

The suffix "-fibroma" is used, when the stroma dominates and occupies an area greater than the cystic portion. The presence of more than 1 cyst over $1 \mathrm{~cm}$ in diameter justifies use of the prefix "cyst". They are further classified into subtypes, according to the histology of the epithelial cells present, as serous, mucinous, endometrioid, clear cell, and mixed categories. The tumor is classified as benign, borderline, or malignant depending upon the degree of epithelial proliferation and its relation to the stromal component of the tumor, although most of the reported ovarian cystadenofibromas were benign. ${ }^{2,5}$
Accurate Preoperative diagnosis cannot be overemphasized as it may prevent patient from undergoing aggressive and radical surgical intervention. But the diagnosis of cystadenofibroma is a difficult one, since they mimic malignant ovarian tumors both macroscopically and on imaging.

On ultrasonography or CT, ovarian cystadenofibroma may have varied appearance. It may present as a complex solid and cystic mass, a solitary cyst, a multiloculated cystic mass, with solid nodules or papillary projections and may show increased vascularity. ${ }^{6}$

In a study done by Cho et al,, all 16 cases of ovarian cystadenofibromas, who presented as complex cystic masses with solid components, were misdiagnosed preoperatively as malignant ovarian neoplasms on CT scan or MRI. ${ }^{1}$

Outwater et al. were the first to report that the solid fibrous component of $\mathrm{z}$, exhibit low-signal intensity on T2-weighted images, later various other reports suggested the same. . $^{1,-10}$

Therefore, MRI may be considered as an imaging modality of choice for diagnosing this tumour since lowsignal intensity due to fibrous component combined with numerous tiny high signal intensity cysts give it characteristic "black sponge" appearance on T2-weighted images. ${ }^{7,9}$

These characteristics on MRI may aid in differentiating it from malignant ovarian tumors which manifest higher intensity on the T2-weighted images and to some extent from other solid benign tumors like fibroma, fibrothecoma and Brenner tumor whose increased amount of collagen and fibrous content, demonstrate low signal on T1W images and very low-signal intensity tissue on the T2W images. ${ }^{10}$ Furthermore, frozen sections may guide appropriate surgical management by establishing precise intraoperative diagnosis of ovarian masses. A correct preoperative diagnosis by MRI and an intraoperative diagnosis by frozen section might save the patient from unnecessary extensive surgery. Hence these modalities may be benefited from, whenever complex ovarian or adnexal lesions are seen on USG. The prognosis of benign cystadenofibroma of ovary is excellent. Recurrence risk is very low on complete removal through surgery.

\section{Funding: No funding sources Conflict of interest: None declared Ethical approval: Not required}

\section{REFERENCES}

1. Cho SM, Byun JY, Rha SE, Jung SE, Park GS, Kim $\mathrm{BK}$, et al. CT and MRI findings of cystadenofibromas of the ovary. Eur Radiol. 2004;14(5):798-804. 
2. Czernobilsky B, Borenstein R, Lancet $\mathrm{M}$. Cystadenofibroma of the ovary. A clinicopathologic study of 34 cases and comparison with serous cystadenoma. Cancer. 1974;34(6):1971-81.

3. Schmidt G, Fowler WC. Ovarian cystadenofibroma in three women with antenatal exposure to diethylstilbestrol. GynecolOncol. 1982;14(2):175-84

4. Wolfe SA, Seckinger DL. Various anatomical types ofovarian adenofibroma. Am J Obstet Gynecol. 1967;99(1):121-5

5. Compton HL, Finck FM. Serous adenofibroma and cystadenofibroma of the ovary. Obstet Gynecol. 1970;36(4):636-45.

6. Alcázar JL, Errasti T, Mínguez JA, Galán MJ, García-Manero M, Ceamanos C. Sonographic features of ovarian cystadenofibromas: spectrum of findings. J Ultrasound Med.2001;20(8):915-19.

7. Outwater EK, Siegelman ES, Talerman A, Dunton C. Ovarian fibromas and cystadenofibrornas: MRI features of the fibrous component. J Mag Resonan Imag. 1997;7(3):465-71.
8. Jung DC, Kim SH, Kim SH. MR imaging findings of ovarian cystadenofibroma and cystadenocarcinofibroma: clues for differential diagnosis. Korean J Radiol. 2006;7(3):199-204.

9. Takeuchi M, Matsuzaki K, Kusaka M, Shimazu H, Yoshida S, Nishitani $\mathrm{H}$, et al. Ovarian cystadenofibromas: Characteristic magnetic resonance findings with pathologic correlation. J Comput Assist Tomogr. 2003;27(6):871-3.

10. Jung SE, Lee JM, RHA SE, Byun JY, Jung JI, Hahn ST. CT and MR Imaging of ovarian tumors with emphasis on differential diagnosis. Radio Graph. 2002;22(6):1305-25.

Cite this article as: Kathuria P, Yadav G, Singh P. The perplexity of malignant ovarian mass in cystadenofibroma: a lesson learnt. Int J Reprod Contracept Obstet Gynecol 2019;8:313-6. 